\title{
The automaticity of perceiving animacy: Goal-directed motion in simple shapes influences visuomotor behavior even when task-irrelevant
}

\author{
Benjamin van Buren ${ }^{1} \cdot$ Stefan Uddenberg $^{1} \cdot$ Brian J. Scholl ${ }^{1}$
}

Published online: 23 November 2015

(C) Psychonomic Society, Inc. 2015

\begin{abstract}
Visual processing recovers not only simple features, such as color and shape, but also seemingly higherlevel properties, such as animacy. Indeed, even abstract geometric shapes are readily perceived as intentional agents when they move in certain ways, and such percepts can dramatically influence behavior. In the wolfpack effect, for example, subjects maneuver a disc around a display in order to avoid several randomly moving darts. When the darts point toward the disc, subjects (falsely) perceive that the darts are chasing them, and this impairs several types of visuomotor performance. Are such effects reflexive, automatic features of visual processing? Or might they instead arise only as contingent strategies in tasks in which subjects must interact with (and thus focus on the features of) such objects? We explored these questions in an especially direct way_-by embedding such displays into the background of a completely independent "foraging" task. Subjects now moved their disc to collect small "food" dots (which appeared sequentially in random locations) as quickly as possible. The darts were task-irrelevant, and subjects were encouraged to ignore them. Nevertheless, foraging was impaired when the randomly moving darts pointed at the subjects' disc, as compared to control conditions in which they were either oriented orthogonally to the subjects' disc or pointed at another moving shape - thereby controlling for nonsocial factors. The perception of animacy thus influences downstream visuomotor behavior in an automatic manner, such that subjects cannot
\end{abstract}

Benjamin van Buren

benjamin.vanburen@yale.edu

1 Department of Psychology, Yale University, Box 208205, New Haven, CT06520-8205, USA completely override the influences of seemingly animate shapes even while attempting to ignore them.

Keywords Social perception · Animacy · Intentionality · Goal-directed motion

Our visual percepts are populated not only by low-level features (such as color and orientation) but also by properties that are more closely associated with higher-level thought (such as animacy and goal-directedness). Perhaps the best demonstration of this is the phenomenon wherein even simple geometric shapes are seen as alive - and as engaged in intentional behaviors - when they move in certain ways (Heider \& Simmel, 1944; Michotte, 1950/1991; for a review, see Scholl \& Tremoulet, 2000). Previous research has identified several of the specific visual cues that give rise to such percepts - such as self-propulsion (e.g., Dasser, Ulbaek, \& Premack, 1989; Pratt, Radulescu, Guo, \& Abrams, 2010), apparent violations of Newtonian mechanics (e.g., Gelman, Durgin, \& Kaufman, 1995; Tremoulet \& Feldman, 2000), and spatiotemporal patterns related to specific forms of intentionality such as chasing (e.g., Gao, Newman, \& Scholl, 2009; Gao \& Scholl, 2011). And such phenomena seem in several ways to be fundamental within visual cognition - appearing early in development (e.g., Gergeley, Nádasdy, Csibra, \& Biró, 1995; Southgate \& Csibra, 2009) and occurring naturally in all observers as a possible cross-cultural universal (Barrett, Todd, Miller, \& Blythe, 2005), except for those with particular neuropsychological impairments, such as autism spectrum disorder (e.g., Klin, 2000; Rutherford, Pennington, \& Rogers, 2006) or amygdala damage (e.g., Heberlein \& Adolphs, 2004). More generally, the perception of animacy seems to reflect visual processing per se (rather than higherlevel interpretations), given that it is resistant to the influences 
of intentions and knowledge, yet is dramatically dependent on subtle display details (for a review, see Scholl \& Gao, 2013).

\section{Consequences of perceiving animacy}

Beyond its rich phenomenology, the perception of animacy also influences downstream processing in various ways. (Here we will seek to emphasize such downstream effects that are themselves highly specific, and perhaps automatic. Of course, visually extracted animacy may also influence a variety of downstream cognitive processes much more generally - consistent with the fact that such displays activate not only regions of the brain devoted to visual processing [e.g., Gao, Scholl, \& McCarthy, 2012, 2015], but also regions associated with higher-level thought and social cognition [e.g., Kuzmanovic et al., 2014; Santos et al., 2010].) Perhaps the most salient example comes from a series of studies on how such cues influence visuomotor performance on various tasks. In one series of experiments (Gao, McCarthy, \& Scholl, 2010), for example, subjects maneuvered a disc around a display filled with randomly moving darts that continuously remained oriented either directly toward the subject's disc (in wolfpack displays) or $90^{\circ}$ away from it (in perpendicular control displays). Despite the objective similarity between these two dynamic patterns, they looked categorically different, with the perpendicular darts being perceived as randomly drifting, and the wolfpack darts perceived (erroneously) as actively pursuing the subject's disc.

The most powerful demonstration of this "wolfpack effect, " however, involved its influence on visuomotor performance in a variant of the so-called Don't Get Caught task (Gao et al., 2010, Exp. 4). When subjects had to actively try to avoid touching the randomly moving darts (and also another "wolf" disc that was objectively pursuing the subject's disc), their evasion was dramatically less successful in the context of wolfpack displays. This result highlights the power of such cues to animacy, since subjects had every incentive to treat the two sorts of displays as equivalent, and not to let the (subtly menacing) wolfpack display impair their performance.

\section{The present study: Irresistible influences of animacy on behavior?}

Although perceiving animacy can influence behavior, the scope and power of such effects remain unclear. In particular, it may be that cues to animacy influence behavior only because the experimental tasks force observers to attend carefully to the putatively animate objects in the first place. Indeed, to our knowledge, the animate shapes have been highly task-relevant in all such experiments to date. In the initial studies that inspired this project, for example, subjects knew while watching the displays that they would have to carefully describe the motions of the objects (Heider \& Simmel, 1944). In many other studies, observers are directly asked to evaluate the perceived animacy of the relevant shapes, by free reports (e.g., Heberlein \& Adolphs, 2004; Klin, 2000), various types of ratings (e.g., Tremoulet \& Feldman, 2000), or explicit categorization (e.g., Barrett et al., 2005). And in the studies of the wolfpack effect, the dart shapes could not have been more task-relevant, since the subjects' goal was explicitly to avoid touching them.

Here, in a strong test of the automaticity of perceived animacy, we asked whether such displays would influence performance even when the putatively animate objects were entirely irrelevant to the task, and while subjects thus attempted to ignore them altogether. In essence, we simply placed such displays into the background of a completely independent task and then told subjects to ignore them.

For the irrelevant "background" display, we used the same sorts of moving darts that were featured in the variant of the Don't Get Caught task described above. We used such displays for two reasons. First, although the wolfpack displays were found to influence performance in several different tasks, their influence was especially profound in these displays (impairing avoidance success by up to $40 \%$, relative to perpendicular control displays). Since any such effects would likely be greatly attenuated when the objects were being ignored, using a display that had previously yielded such a large effect magnitude seemed wise. Second, this particular display allowed for another especially powerful sort of control, beyond the perpendicular displays. In addition to being oriented toward the subject's disc (which, as noted, greatly impaired performance), the darts could remain oriented toward a different (computer-controlled) disc (which led to no impairment at all). This control effectively ensured that the impairments derived only from the social significance of the display, and not from any other, lower-level factors (Gao et al., 2010). For example, one might worry that the darts' locations were accurately perceived in the perpendicular configuration, but imperfectly perceived in a wolfpack configuration - but this would be true for both sorts of wolfpack displays, only one of which impaired performance. Similarly, one might worry that the impairments were due to some novel form of grouping or attentional capture based on coordinated orientations - but of course, such coordination would be equated, regardless of whether the wolfpack was pointing at you (in which case it would impair performance) or at another object (in which case it wouldn't).

Subjects performed a simple "foraging" task, in which they maneuvered a disc to pick up sequentially appearing "food" dots while several shapes moved randomly in the background (see Fig. 1). These background shapes always consisted of 13 darts and a circle, and subjects were explicitly told to simply 


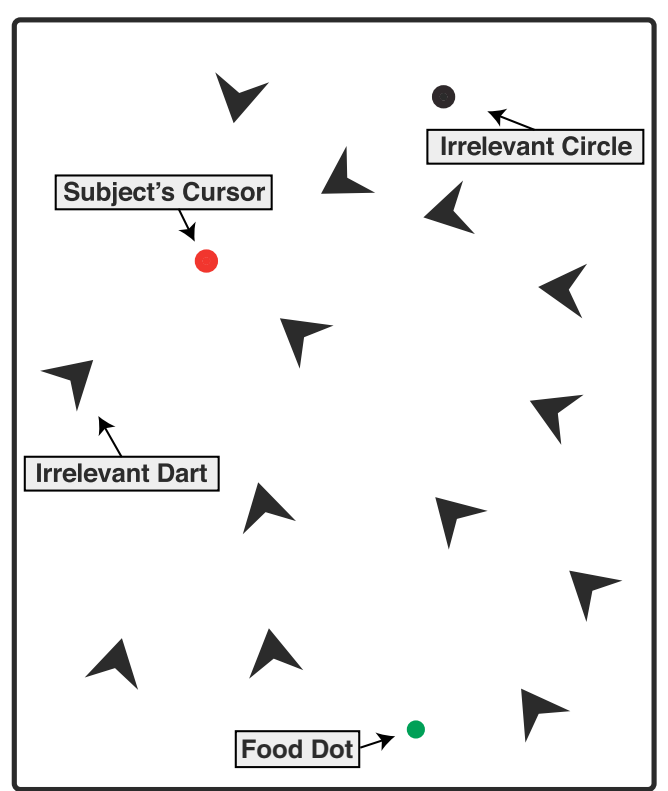

Fig. 1 A cartoon depiction of a still frame from a subject-directed wolfpack trial. Subjects used the computer mouse to move a foraging disc (depicted in red, and labeled 'Subject's Cursor') to collect sequentially appearing food dots (depicted in green, and labeled 'Food Dot'), while a circle and 13 darts (black) moved haphazardly in the background

ignore them in order to perform the foraging task as quickly as possible. On subject-directed wolfpack trials, the darts always pointed toward the subject's foraging disc as they moved randomly (Fig. 2A). On perpendicular-to-subject trials, the darts always pointed orthogonally to the subject's foraging disc (Fig. 2B). And on other-directed wolfpack trials, the darts always pointed at the randomly moving (computercontrolled) circle (Fig. 2C). These conditions are difficult to depict in static figures, but dynamic animations can be viewed online at www.yale.edu/perception/foraging/ .

If this form of perceived animacy influences performance only when task demands require subjects to attend carefully to the darts, then we might observe no effect here, in contrast to previous results (Gao et al., 2010). But, if the influence of

\section{Trial Types}

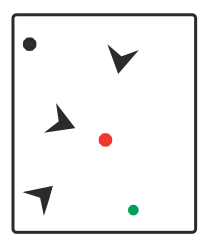

(A)

Subject-

Directed

Wolfpack

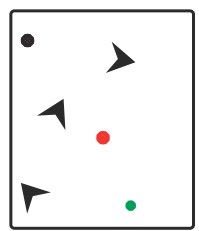

(B)

Motion Control: Perp. to Subject

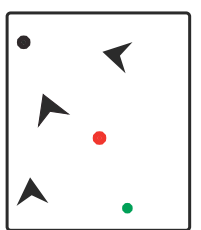

(C)

Pointing Control: Wolfpack Other-Directed

Fig. 2 Illustration of the three trial types (see the text for details). The subject's foraging disc is depicted as red in the electronic version of the paper, the computer-controlled circle is depicted in black, and a smaller food dot is depicted as green in the electronic version perceived animacy on this form of visuomotor behavior is truly automatic and irresistible, then we might observe some influence of the wolfpack even when the shapes are irrelevant and when subjects are trying to ignore them.

\section{Method}

\section{Subjects}

A total of 40 naïve subjects ( 20 male, 20 female) from the Yale University and New Haven communities (average age $=19.1$ years, $S D=0.92$ years), all with normal or correctedto-normal visual acuity, participated in exchange for course credit or a monetary payment. This sample size was chosen on the basis of a power analysis on pilot data.

\section{Apparatus}

Stimuli were presented via a Macintosh computer on a 22in. Dell LCD display, using custom software written in Python with the PsychoPy libraries (Peirce, 2007). Subjects sat in a dimly lit room without restraint approximately $60 \mathrm{~cm}$ away from the functional part of the display, which subtended approximately $11.30^{\circ} \times 11.30^{\circ}$ in the center of the monitor, and all subsequent measurements are based on this viewing distance.

\section{Stimuli}

The functional part of the display had a black background and was bounded by a centered one-pixel $\left(0.03^{\circ}\right)$ white outlined square. The subject controlled the movements of a white disc (diameter $\left.0.22^{\circ}\right)$ surrounded by a one-pixel $\left(0.03^{\circ}\right)$ black outline. The "food" dots were each smaller white discs (diameter $0.11^{\circ}$ ) surrounded by the same type of outline. (Given the black background, these outlines were only visible when the disc in question intersected another object; since the outlines were always drawn in front during any intersection, these discs were thus visible at all times.) The randomly moving computer-controlled circle was a slightly larger white disc (diameter $0.27^{\circ}$ ). The darts were each derived from equilateral triangles whose "nose" and right and left "wings" sat on the perimeter of an invisible $1.18^{\circ}$ diameter circle (see Fig. 1).

The computer-controlled circle and the darts each had an effective heading (initially randomly determined) that controlled the direction of a constant $3.24 \%$ s displacement. The animations were presented at 30 frames/s. This heading itself was constantly changing, with this rate of change being an initial random choice between $-9.32^{\circ}$ and $9.32^{\circ}$. Subsequently, on each frame this rate of heading change itself had a $1 / 3$ chance of being updated by a randomly chosen value between $-9.32^{\circ}$ and $9.32^{\circ}$. This led to smooth haphazard trajectories 
for each of these objects. Whenever one of these objects reached the bounds of the display, however, the horizontal or vertical component of its velocity was reversed, so that it appeared to "bounce" off of the edges. (The subject's foraging disc was also confined within these bounds, but was otherwise simply controlled by the computer mouse, limited only by the physical constraints of the motions themselves.)

On subject-directed wolfpack trials, the darts were always oriented toward the subject's foraging disc. On perpendicularto-subject trials, the darts were always oriented orthogonally to the subject's foraging disc. And on other-directed wolfpack trials, the darts were always oriented toward the computercontrolled circle.

\section{Procedure}

Each trial began with the subject using the computer mouse to move his or her foraging disc into a rectangular $\left(1.35^{\circ} \times\right.$ $0.68^{\circ}$ ) gray box, labeled "start", at the center of the display. The gray box then disappeared, and all of the other stimuli-a single food dot, the computer-controlled circle, and 13 dartsimmediately appeared in randomly chosen locations (with the food dot being constrained to appear at least $6.07^{\circ}$ away from the subject-controlled foraging disc), after which the computer-controlled circle and the darts immediately began moving.

Subjects proceeded to "collect" food dots by moving their foraging disc over them. They were instructed to do this as quickly as possible and to simply ignore all of the other moving shapes. As soon as the subject's foraging disc touched the food dot, a new food dot appeared in a random location at least $6.07^{\circ}$ away. A "score" was always visible at the top of the display, presented as an integer, starting with a value of 0 (drawn in Calibri with a height of $0.43^{\circ}$, horizontally centered, with its lowest contour separated by a gap of $0.35^{\circ}$ from the top border of the white square). Whenever a subject collected a food dot, the score increased by 100 . A trial continued until a subject had collected a 10 th food dot (i.e., to reach a score of 1 , 000), at which point all of the objects disappeared and were replaced by the subject-controlled foraging disc (vertically centered at the far left of the functional part of the display) and the central gray "start box"-with the next trial beginning as soon as the subject's foraging disc touched the start box.

\section{Design}

Subjects first completed six practice trials, consisting of two trials for each of the three conditions, presented in a different random order for each subject. They then completed 24 experimental trials - eight each of the three conditions, presented in a different random order for each subject (and further constrained to ensure that equal numbers of trials of each condition type were presented in each half of the experiment).
A written prompt encouraged subjects to take a short selftimed break at the halfway point (i.e., after the 12th trial). Each trial lasted approximately $14 \mathrm{~s}$. On each experimental trial, we recorded the time taken to forage for the ten food dots, the subject's average speed, and the locations of the objects on each frame.

\section{Results}

The average foraging time per trial is depicted in Fig. 3A for each of the three condition types. Inspection of this figure suggests a simple pattern: Foraging times were longer for the subject-directed wolfpack trials, relative to both the perpendicular-to-subject and other-directed wolfpack trials (which did not themselves differ). These impressions were verified by the following statistical analysis. A one-way repeated measures analysis of variance (ANOVA) on foraging times revealed a marginally significant effect of dart orientation $[F(1$, $\left.39)=2.85, p=.064, \eta_{\mathrm{p}}{ }^{2}=.068\right]$. Planned comparisons then revealed that subjects took longer to forage for food dots on subject-directed wolfpack trials than on either perpendicular-tosubject trials $[t(39)=2.15, p=.038, d=0.34]$ or other-directed wolfpack trials $[t(39)=2.14, p=.039, d=0.34]$, which did not themselves differ $[t(39)=0.05, p=.962, d=0.01]$.

What explains this difference in foraging times? One obvious possibility is that subjects traversed less direct paths to the food dots in the subject-directed wolfpack trials, perhaps because they felt threatened by — and thus wanted to avoid - the darts. To explore this possibility, we broke the trials down into individual "food runs." For each food run, we generated the most efficient path (i.e., the straight line) between the subject's location at the start of the run and the next food dot. We then measured the maximum perpendicular distance between this line and the subject's trajectory (the maximum deviation). A one-way repeated measures ANOVA on these maximum deviations, however, revealed no effect of condition $[F(1$, $\left.39)=0.245, p=.783, \eta_{\mathrm{p}}{ }^{2}=.006\right]$, and none of the individual conditions differed from the others $(t \mathrm{~s} \leq 0.68, p \mathrm{~s} \geq$ $.50, d \mathrm{~s} \leq 0.11){ }^{1}$

If the subject did not forage on less efficient paths in the subject-directed wolfpack condition, it follows that the greater foraging time must be explained by appeal to temporal factors alone - and the most obvious such factor is the subject's average foraging speed. And indeed, a one-way repeated measures ANOVA on average foraging speed (measured simply

\footnotetext{
${ }^{1}$ Similar null effects were observed for all other related such "spatial" analyses that we computed, including (1) area under the curve relative to the most efficient path, (2) brute excess distance relative to the length of the most efficient path, (3) the average distance at all moments between the subject-controlled disc and the darts, (4) the average distance between the subject-controlled disc and the nearest dart, and (5) the number of times the subject's foraging disc made contact with the darts.
} 
(A) Initial Experiment

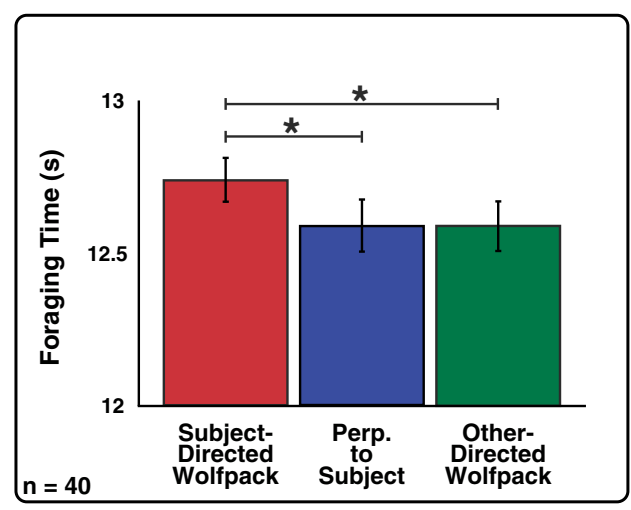

(B) Direct Replication

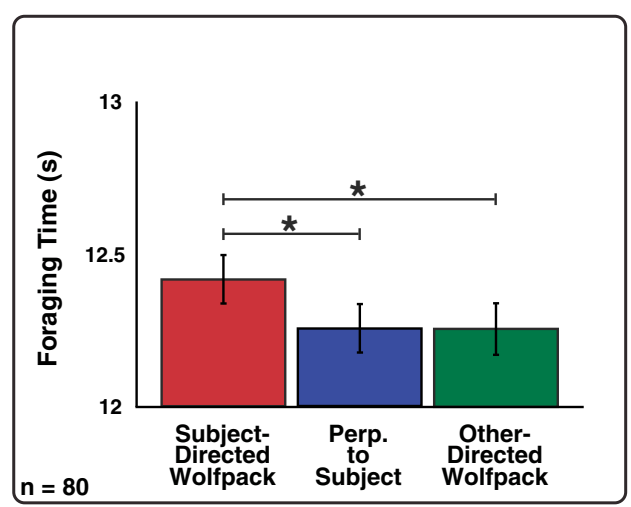

Fig. 3 Average foraging times per trial in both (A) the initial experiment and (B) the direct replication. In both experiments, subjects took longer to forage for dots when the darts pointed at their foraging disc, relative to the control conditions. Error bars are $95 \%$ confidence intervals

as the total distance traveled divided by the total time taken) revealed a significant effect of condition $[F(1,39)=7.79, p<$ $\left..001, \eta_{\mathrm{p}}^{2}=.166\right]$. Planned comparisons revealed that speeds were slower on subject-directed wolfpack trials than on either perpendicular-to-subject trials $(7.07 \%$ vs. $7.22 \%$ s $)$ $[t(39)=3.66, p<.001, d=0.58]$ or other-directed wolfpack trials $\left(7.15^{\circ} / \mathrm{s}\right)[t(39)=2.41, p=.021, d=$ 0.38 ] - and that speeds were marginally faster on perpendicular-to-subject trials than on other-directed wolfpack trials $[t(39)=1.74, p=.089, d=0.28]$.

\section{Direct replication}

Given that the darts were completely irrelevant to the subjects' task and that subjects were thus explicitly instructed to ignore them, it is perhaps not a surprise that the key differences reported above - though all statistically significant - were also very small (comprising just a few seconds over the course of the experiment). As such - and also because this experimental paradigm was developed for the present project (and so had never been used before) - it seemed prudent to run a direct replication. A power analysis on the primary data reported above indicated that we would need 80 subjects to replicate the observed effects from the main experiment with $80 \%$ power. We therefore recruited 80 new subjects (45 female, 35 male; average age $=21.0$ years, $S D=3.30$ years) and replicated the experiment exactly.

The resulting foraging times are depicted in Fig. 3B. Inspection of this graph reveals that this replication produced the same pattern of results, and this was verified via the same statistical analyses. The main effect of condition on foraging times was significant $\left[F(1,79)=3.56, p=.031, \eta_{\mathrm{p}}{ }^{2}=.043\right]$, and again, subjects took longer to forage on subject-directed wolfpack trials than on either perpendicular-to-subject trials $[t(79)=2.37, p=.020, d=0.26]$ or other-directed wolfpack trials $[t(79)=2.23, p=.029, d=0.25]$, which did not themselves differ $[t(79)=0.074, p=.941, d=0.01]$. There was no effect of condition on either the maximum deviations $[F(1,79)$ $\left.=0.36, p=.701, \eta_{\mathrm{p}}{ }^{2}=.004\right]$ or foraging speed $[F(1,79)=$ $\left.2.19, p=.115, \eta_{\mathrm{p}}{ }^{2}=.027\right]$, however, and the only individual comparison that was (barely) reliable was that foraging speeds were slower on subject-directed wolfpack trials than on perpendicular-to-subject trials $(7.41 \%$ vs. $7.49 \%)[t(79)=$ $1.99, p<.05, d=0.22$; all other $t \mathrm{~s} \leq 1.31$, all other $p \mathrm{~s} \geq$ .194 , all other $d \mathrm{~s} \leq 0.15]$.

\section{Discussion}

The primary result of this study was extremely clear: Subjects took longer to forage for food dots when the irrelevant background darts were always pointing at (vs. $90^{\circ}$ away from) the disc they were controlling. This was not due to the rotational motion that was required to keep the darts oriented, since that was equated across these conditions. And it was not due to any other displaywide effect of such coordinated orientations (e.g., related to grouping or misperceived locations), since such stimulus factors were equated across the two wolfpack conditions. Instead, it appears that this result must reflect the perceived animacy and the social significance that resulted from having the darts point at the subjects themselves. Just how such effects arise from particular patterns of behavior (as when subjects are controlling their foraging disc), however, remains elusive, and will require more investigation. We saw some hint that foraging speed might be the culprit, since subjects tended to move their disc more slowly when the darts were pointing at them (perhaps due to a type of "freeze response" related to threatening stimuli; e.g., Stins \& Beek, 2007). But this effect was clearly not as strong or reliable as the primary effect of foraging time.

It bears repeating that the magnitudes of the key effects were all very small — a few seconds' difference at best, over the entire experiment. These small differences, however, were reliable, and they replicated in a second sample. And, of 
course, they also conceptually replicated the results of the modified Don't Get Caught task from the initial demonstration of the "wolfpack effect" (Exp. 4, Gao et al., 2010): In that experiment, the subject-directed wolfpack condition was uniquely detrimental to avoidance behavior when the darts were extremely task-relevant - just as here, where this condition was uniquely detrimental to the time taken to complete our novel foraging task when the darts were entirely taskirrelevant. In general, we remain impressed and slightly amazed that such effects existed at all, given that they were driven only by aspects of "background" stimuli that subjects knew were irrelevant and were actively trying to ignore. That they could not do so indicates that the perception of animacy - and its influence on downstream visuomotor behavior - is not isolated to those situations in which subjects are motivated to attend to the relevant items (as has been the case in all previous experiments). Instead, the perception of animacy appears to influence behavior automatically, such that subjects cannot completely override the influences of seemingly animate shapes even while attempting to ignore them.

Author note For helpful conversation and/or comments on previous drafts, we thank Tao Gao, Greg McCarthy, Jamie McPartland, and the members of the Yale Perception \& Cognition Laboratory. This project was funded by National Science Foundation Graduate Research Fellowships awarded to both B.v.B. and S.U.

\section{References}

Barrett, H., Todd, P., Miller, F., \& Blythe, M. (2005). Accurate judgments of intention from motion cues alone: A cross-cultural study. Evolution and Human Behavior, 26, 313-331.

Dasser, V., Ulbaek, I., \& Premack, D. (1989). The perception of intention. Science, 243, 365-367.

Gao, T., McCarthy, G., \& Scholl, B. J. (2010). The wolfpack effect: Perception of animacy irresistibly influences interactive behavior. Psychological Science, 21, 1845-1853. doi:10.1177/ 0956797610388814

Gao, T., Newman, G. E., \& Scholl, B. J. (2009). The psychophysics of chasing: A case study in the perception of animacy. Cognitive Psychology, 59, 154-179.

Gao, T., \& Scholl, B. J. (2011). Chasing vs. stalking: Interrupting the perception of animacy. Journal of Experimental Psychology: Human Perception and Performance, 37, 669-684. doi:10.1037/ a0020735

Gao, T., Scholl, B. J., \& McCarthy, G. (2012). Dissociating the detection of intentionality from animacy in the right posterior superior temporal sulcus. Journal of Neuroscience, 32, 14276-14280. doi:10.1523/ JNEUROSCI.0562-12.2012

Gao, T., Scholl, B. J., \& McCarthy, G. (2015). The perception of animacy pervades visual processing: Selective engagement of cortical regions associated with faces and motion. Manuscript submitted for publication.

Gelman, R., Durgin, F., \& Kaufman, L. (1995). Distinguishing between animates and inanimates: Not by motion alone. In D. Sperber, D. Premack, \& A. J. Premack (Eds.), Causal cognition: A multidisciplinary debate (pp. 150-184). Oxford: Oxford University Press, Clarendon Press.

Gergeley, G., Nádasdy, Z., Csibra, G., \& Biró, S. (1995). Taking the intentional stance at 12 months of age. Cognition, 56, 165-193. doi:10.1016/0010-0277(95)00661-H

Heberlein, A. S., \& Adolphs, R. (2004). Impaired spontaneous anthropomorphizing despite intact perception and social knowledge. Proceedings of the National Academy of Sciences, 101, 74877491. doi:10.1073/pnas.0308220101

Heider, F., \& Simmel, M. (1944). An experimental study of apparent behavior. American Journal of Psychology, 57, 243-259.

Klin, A. (2000). Attributing social meaning to ambiguous visual stimuli in higher functioning autism and Asperger syndrome: The social attribution task. Journal of Child Psychology and Psychiatry, 41, 831-846.

Kuzmanovic, B., Schilbach, L., Georgescu, A., Kockler, H., Santos, N., Shah, N., ... Vogeley, K. (2014). Dissociating animacy processing in high-functioning autism: Neural correlates of stimulus properties and subjective ratings. Social Neuroscience, 9, 309-325.

Michotte, A. (1991). The emotions regarded as functional connections. In G. Thinès, A. Costall, \& G. Butterworth (Eds.), Michotte's experimental phenomenology of perception (pp. 103-116). Hillsdale, NJ: Erlbaum. [Reprinted from M. Reymert (Ed.). (1950). Feelings and emotions: The Mooseheart symposium (pp. 114-125). New York, NY: McGraw-Hill]

Peirce, J. W. (2007). PsychoPy-Psychophysics software in Python. Journal of Neuroscience Methods, 162, 8-13. doi:10.1016/j. jneumeth.2006.11.017

Pratt, J., Radulescu, P. V., Guo, R. M., \& Abrams, R. A. (2010). It's alive! Animate motion captures attention. Psychological Science, 21, 1724-1730.

Rutherford, M., Pennington, B., \& Rogers, S. (2006). The perception of animacy in young children with autism. Journal of Autism and Developmental Disorders, 36, 983-992.

Santos, N. S., Kuzmanovic, B., David, N., Rotarska-Jagiela, A., Eickhoff, S. B., Shah, J. N., ... Vogeley, K. (2010). Animated brain: A functional neuroimaging study on animacy experience. NeuroImage, 53, 291-302. doi:10.1016/j.neuroimage.2010.05.080

Scholl, B. J., \& Gao, T. (2013). Perceiving animacy and intentionality: Visual processing or higher-level judgment? In M. D. Rutherford \& V. A. Kuhlmeier (Eds.), Social perception: Detection and interpretation of animacy, agency, and intention (pp. 197-230). Cambridge: MIT Press.

Scholl, B. J., \& Tremoulet, P. D. (2000). Perceptual causality and animacy. Trends in Cognitive Sciences, 4, 299-309. doi:10.1016/ S1364-6613(00)01506-0

Southgate, V., \& Csibra, G. (2009). Inferring the outcome of an ongoing novel action at 13 months. Developmental Psychology, 45, 1794 1798. doi:10.1037/a0017197

Stins, J. F., \& Beek, P. J. (2007). Effects of affective picture viewing on postural control. BMC Neuroscience, 8(83), 1-7. doi:10.1186/14712202-8-83

Tremoulet, P. D., \& Feldman, J. (2000). Perception of animacy from the motion of a single object. Perception, 29, 943-951. 